\title{
Oversampled Deadbeat Current Control Strategy for PMSM Drives
}

\author{
Luca Rovere, Andrea Formentini, Pericle Zanchetta \\ Department of Electrical and Electronic Engineering, The University of Nottingham \\ Nottingham. NG7 2RD, U.K. \\ luca.rovere@nottingham.ac.uk, andrea.formentini@nottingham.ac.uk,pericle.zanchetta@nottingham.ac.uk
}

\begin{abstract}
This paper presents a novel deadbeat current control approach for Permanent Magnet Synchronous Motors (PMSMs) drives capable of operating at a controller sampling frequency multiple of the power converter switching frequency. The proposed technique permits to achieve a constant switching frequency and an optimal current ripple along with a high current loop bandwidth and robust behaviour to parameter variation.
\end{abstract}

\section{Keywords—Deadbeat Control, PMSM Drives}

\section{INTRODUCTION}

PMSMs offer different advantages in the field of variable-speed AC drives compared to other motor types such as induction motors. They present higher efficiency, higher performance, compact construction, higher torque per volume ratio. Recently the trend towards a fully digital control of power converters based on deadbeat current control is increasing [1-3]. This technique permits to achieve a fast transient response, precise current control and it is fully compatible with digital control platforms. The deadbeat control in combination with the space vector modulation (SVM) technique is able to provide the lowest distortion and the lowest current ripples [4]. In the attempt of pushing the system dynamic performance without increasing the converter switching frequency and/or using expensive new Wide Band Gap (WBG) devices, another emerging model based control technique, Finite Control Set Model Predictive Control (FCSMPC) [5-8], has received in recent years a great interest from the academic community. It uses the model of the system to predict its future states for every possible control action. The best control action is then chosen by minimizing a cost function. This approach does not require a modulator, reduces the control sample time and decreases consequently the response time. These features lead to high bandwidth in the dynamic response and robust behaviour to parameter variation and system imperfection. On the other hand however, the absence of a modulator results in a variable switching frequency that usually results in a suboptimal current ripple. This paper investigates a new dead beat approach in order to operate at an increased controller sampling frequency without increasing the commutation frequency of the power converter. The proposed technique permits to achieve a constant switching frequency and an optimal current ripple along with a high current loop bandwidth and robust behaviour to parameter variation.

The system configuration studied here is composed by a DC voltage source that supplies a three phase two levels Voltage Source Inverter (VSI) which is connected to a PMSM. The proposed new deadbeat current controller generates the phase voltage control references which are fed into a Pulse Width Modulator (PWM) that gives as output the gate signals to control the VSI IGBTs. The new oversampled deadbeat controller is obtained by discretizing the system equations over a variable sample time $T_{s_{i}}$ resulting in an increased control sampling frequency. Although the proposed oversampling approach increase the robustness to machine parameters variation, on the other hand it introduces a distortion in the control voltages fed into the PWM, causing an offset in the controlled PMSM currents. To eliminate this behaviour a compensation strategy has been developed and successfully validated. Simulations have been carried out with successful results regarding the robustness achieved with the oversampling algorithm and the elimination of the control offset with the control voltage compensation. Furthermore increased performance at speed higher than $3000 \mathrm{rpm}$, consisting in the phase current Total Harmonic Distortion (THD) reduction, has been noticed with the compensation proposed.

\section{PMSM MODEL AND DEADBEAT CONTROL}

The mathematical model of a PMSM in rotor synchronous reference frame qd is reported in (1):

$$
\left[\begin{array}{c}
V_{q s} \\
V_{d s}
\end{array}\right]=\left[\begin{array}{cc}
R_{s} & 0 \\
0 & R_{s}
\end{array}\right]\left[\begin{array}{l}
i_{q s} \\
i_{d s}
\end{array}\right]+\left[\begin{array}{cc}
p & \omega_{r} \\
-\omega_{r} & p
\end{array}\right]\left[\begin{array}{l}
\lambda_{q s} \\
\lambda_{d s}
\end{array}\right]
$$

where $V_{q s}, V_{d s}, \lambda_{q s}, \lambda_{d s}$ and $i_{q s}, i_{d s}$ are the stator q- and daxes voltage, flux linkage, and current in the rotor reference frame, $R_{s}$ is the stator resistance, $\omega_{r}$ is the rotor angular velocity, and $\mathrm{p}$ is the derivative operator. The stator flux linkage viewed in a rotor synchronous reference frame is given by (2):

where

$$
\left[\begin{array}{l}
\lambda_{q s} \\
\lambda_{d s}
\end{array}\right]=\left[L_{s s}^{q d}\right]\left[\begin{array}{l}
i_{q s} \\
i_{d s}
\end{array}\right]+\left[\lambda_{m}^{q d}\right]
$$




$$
\left[L_{s s}^{q d}\right]=\left[\begin{array}{cc}
L_{q} & 0 \\
0 & L_{d}
\end{array}\right] \quad\left[\lambda_{m}^{q d}\right]=\lambda_{m}\left[\begin{array}{l}
0 \\
1
\end{array}\right]
$$

$L_{q}, L_{d}$ are q-axis and d-axis inductance in the rotor reference frame, $\lambda_{m}$ is the peak flux linkage established by the magnets. The model in (1) has been discretized using the Euler discretization method for digital implementation. The discretization around a generic time instant $k$ leads to the following equations:

$$
\begin{gathered}
{\left[\begin{array}{l}
i_{q s k+1} \\
i_{d s k+1}
\end{array}\right]=A\left[\begin{array}{l}
i_{q s k} \\
i_{d s k}
\end{array}\right]+B_{1}\left[\begin{array}{c}
V_{q s k} \\
V_{d s k}
\end{array}\right]+B_{2}\left[\begin{array}{c}
\lambda_{m} \\
0
\end{array}\right]} \\
A=\left[\begin{array}{cc}
1-\frac{R_{s}}{L_{q}} T_{s} & -\omega_{r} \frac{L_{d}}{L_{q}} T_{s} \\
\omega_{r} \frac{L_{q}}{L_{d}} T_{s} & 1-\frac{R_{s}}{L_{d}} T_{s}
\end{array}\right] \quad B_{1}=\left[\begin{array}{cc}
\frac{T_{s}}{L_{q}} & 0 \\
0 & \frac{T_{s}}{L_{d}}
\end{array}\right] \\
B_{2}=\left[\begin{array}{cc}
-\frac{T_{s} \omega_{r}}{L_{q}} & 0 \\
0 & 0
\end{array}\right]
\end{gathered}
$$

Even if the machine equations are presented considering $L_{q}$ and $L_{d}$, in the following the motor has been considered without any anisotropy, that means $L_{q}=L_{d}=L$.

The equations of the classical Deadbeat control for the PMSM can be obtained from (4) substituting to the $\mathrm{k}+1$ currents values the reference ones and solving for the voltages:

$$
\left[\begin{array}{c}
V_{q s_{k}} \\
V_{d s_{k}}
\end{array}\right]=B_{1}^{-1}\left\{\left[\begin{array}{c}
i_{q s_{k}}{ }^{r e f} \\
i_{d s_{k}} \\
r e f
\end{array}\right]-A\left[\begin{array}{c}
i_{q s_{k}} \\
i_{d s_{k}}
\end{array}\right]-B_{2}\left[\begin{array}{c}
\lambda_{m} \\
0
\end{array}\right]\right\}
$$

The resulting control action permits, if no saturation occurs, to reach the current reference value in one sample time. The control voltages obtained are then transformed from the synchronous reference frame to the abc phase coordinates. These signals are finally saturated and injected in a modulator that gives as output the gate signals from the comparison of the control voltages with a triangular waveform carrier of period $T_{c}$. Fig. 1 shows the overall control structure.

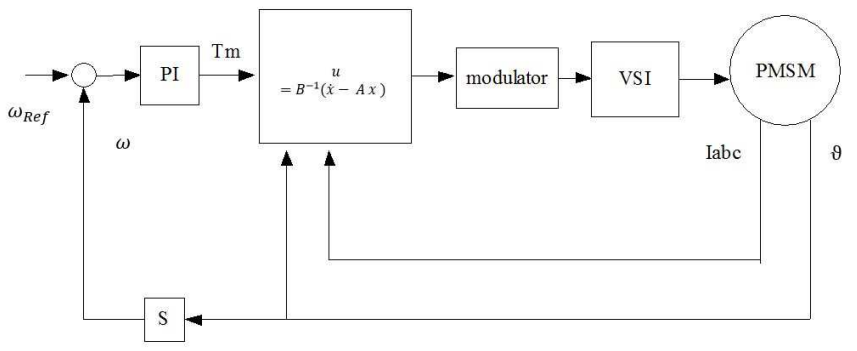

Fig. 1 - Complete control structure

\section{OVERSAMPLED DEADBEAT APPROACH}

In order to increase the controller sampling time $T_{x}$ without changing the switching frequency (i.e. the modulator carrier frequency) some consideration should be done. If the control approach described earlier is simply implemented to a higher frequency, the system becomes quickly unstable as the ratio $\frac{T_{x}}{T_{C}}$ decreases due to the interaction with the modulator. The control voltages (6) in fact, are optimal only if they are equal to the inverter output voltages averaged on a $T_{x}$ period. Using a modulator however, it is guaranteed only on a carrier semiperiod, i.e. $\frac{T_{c}}{2}$. To overcome this problem, at each controller sample time the system computes the optimal control action to reach the current reference at the end of the carrier segment as depicted in Fig. 2. System (6) has been rewritten as

$$
\begin{aligned}
& {\left[\begin{array}{c}
i_{q s_{k+n_{c}-1}} \\
i_{d s_{k+n_{c}-1}}
\end{array}\right]=A_{T_{s_{i}}}\left[\begin{array}{c}
i_{q s_{k}} \\
i_{d s_{k}}
\end{array}\right]+B_{1 T_{s_{i}}}\left[\begin{array}{c}
V_{q s_{k}} \\
V_{d s_{k}}
\end{array}\right]+B_{2 T_{s_{i}}}\left[\begin{array}{c}
\lambda_{m} \\
0
\end{array}\right]} \\
& i=0,1, \ldots, n_{c}-1
\end{aligned}
$$

where $n_{c}$ has been defined as 2 times the ratio between the carrier period $T_{c}$ and the control period $T_{x}$. The matrix subscripts indicate the time used in the discretization process and are defined as

$$
T_{s_{i}}=\frac{T_{c}}{2}-i T_{x}=\left(n_{c}-i\right) T_{x}, \quad i=0,1, \ldots, n_{c}-1
$$

$i$ is reset to 0 every $\frac{T_{C}}{2}$, i.e. at the end of each carrier segment. (7) can then be solved for the control action substituting to the left hand side the current references, resulting in

$$
\left[\begin{array}{c}
V_{q s_{k}} \\
V_{d s_{k}}
\end{array}\right]=B_{1 T_{s_{i}}}^{-1}\left\{\left[\begin{array}{l}
i_{q s_{k}}{ }^{r e f} \\
i_{d s_{k}} \\
\text { ref }
\end{array}\right]-A_{T_{s i}}\left[\begin{array}{c}
i_{q s_{k}} \\
i_{d s_{k}}
\end{array}\right]-B_{2 T_{s_{i}}}\left[\begin{array}{c}
\lambda_{m} \\
0
\end{array}\right]\right\}
$$

Please note that the only difference between (6) and (9) is that the latter is computed every $T_{x}$ with a different set of matrices. Note also that the $n_{c}$ set of matrices can be precomputed and stored in memory.

If during a carrier semi period the current reference doesn't change, the model parameters are correct and the actual applied voltages match with the computed one, (9) returns a set of $n_{c}$ equal control action for the whole semi period. In practice however, the voltages calculated are not applied until there is an intersection between one of the phase control voltages and the carrier. This results in a voltage references distortion that can compromise the system performances. A compensation method is proposed in the following to solve this problem.

The machine currents at the time instant $\mathrm{k}$ can be written starting from the previous time instant $\mathrm{k}-1$ distant $T_{x}$ as

$$
\left[\begin{array}{l}
i_{q s_{k}} \\
i_{d s_{k}}
\end{array}\right]=A_{T_{x}}\left[\begin{array}{c}
i_{q s_{k-1}} \\
i_{d s_{k-1}}
\end{array}\right]+B_{1 T_{x}}\left[\begin{array}{c}
V_{q s_{k-1}} \\
V_{d s_{k-1}}
\end{array}\right]+B_{2 T_{x}}\left[\begin{array}{c}
\lambda_{m} \\
0
\end{array}\right]
$$

Substituting (10) in (9) 


$$
\begin{gathered}
{\left[\begin{array}{c}
V_{q s_{k}} \\
V_{d s_{k}}
\end{array}\right]=B_{1}^{-1}{ }_{T_{s_{i}}}^{-1}\left[\left[\begin{array}{cc}
i_{q s_{k}}{ }^{r e f} \\
i_{d s_{k}} & r e f
\end{array}\right]\right.} \\
-A_{T_{s_{i}}}\left[A_{T_{x}}\left[\begin{array}{c}
i_{q s_{k-1}} \\
i_{d s_{k-1}}
\end{array}\right]+B_{1 T_{x}}\left[\begin{array}{c}
V_{q s_{k-1}} \\
V_{d s_{k-1}}
\end{array}\right]\right. \\
\left.\left.+B_{2 T_{x}}\left[\begin{array}{c}
\lambda_{m} \\
0
\end{array}\right]\right]-B_{2}\left[\begin{array}{c}
\lambda_{m} \\
0
\end{array}\right]\right\}
\end{gathered}
$$

In order to obtain the compensation term, (11) is written two times. Firstly, equation (11a) is obtained by substituting to $V_{q s_{k-1}}$ and $V_{d s_{k-1}}$ the control voltages calculated by the deadbeat controller equations (9) at the time instant $\mathrm{k}-1$ that we will call $V_{q s_{k-1}}^{c}$ and $V_{d s_{k-1}^{c}}$.

$$
\begin{aligned}
& {\left[\begin{array}{c}
V_{q s_{k}}{ }^{c} \\
V_{d s_{k}}{ }^{c}
\end{array}\right]=B_{1}^{-1}\left\{\left[\begin{array}{l}
i_{T_{i}} \\
i_{q s_{k}}{ }^{r e f} \\
i_{d s_{k}}
\end{array}\right]\right.} \\
& -A_{T_{s_{i}}}\left[A_{T_{x}}\left[\begin{array}{c}
i_{q s_{k-1}} \\
i_{d s_{k-1}}
\end{array}\right]+B_{1 T_{x}}\left[\begin{array}{c}
V_{q s_{k-1}}{ }^{c} \\
V_{d s_{k-1}}^{c}
\end{array}\right]\right. \\
& \left.\left.+B_{2 T_{x}}\left[\begin{array}{c}
\lambda_{m} \\
0
\end{array}\right]\right]-B_{2 T_{s_{i}}}\left[\begin{array}{c}
\lambda_{m} \\
0
\end{array}\right]\right\}
\end{aligned}
$$

Secondly, (11) is written substituting to $V_{q s_{k-1}}$ and $V_{d s_{k-1}}$ the real voltages applied by the inverter $V_{q s_{k-1}}^{r}$ and $V_{d s_{k-1}}^{r}$ obtaining (11b).

$$
\begin{aligned}
& {\left[\begin{array}{c}
V_{q s_{k}}^{r} \\
V_{d s_{k}}{ }^{r}
\end{array}\right]=B_{1_{T_{s_{i}}}^{-1}}^{-1}\left\{\left[\begin{array}{l}
i_{q s_{k}} \\
i_{d s_{k}} \\
i^{e f f}
\end{array}\right]\right.} \\
& -A_{T_{S i}}\left[A_{T_{x}}\left[\begin{array}{l}
i_{q s_{k-1}} \\
i_{d s_{k-1}}
\end{array}\right]+B_{1 T_{x}}\left[\begin{array}{c}
V_{q s_{k-1}}^{r} \\
V_{d s_{k-1}}^{r}
\end{array}\right]\right. \\
& \left.\left.+B_{2 T_{x}}\left[\begin{array}{c}
\lambda_{m} \\
0
\end{array}\right]\right]-B_{2 T_{s_{i}}}\left[\begin{array}{c}
\lambda_{m} \\
0
\end{array}\right]\right\}
\end{aligned}
$$

The compensation term permits to obtain $V_{q s_{k-1}}{ }^{c}$ equal to $V_{q s_{k}}{ }_{k}$ and $V_{d s_{k-1}^{c}}^{c}$ equal to $V_{d s_{k}}^{c}$ hence for the q- and d-axes $c o m p_{q} i$ and $\operatorname{comp}_{d i}$ are obtained by subtracting (11b) to (11a) resulting in (12)

$$
\begin{gathered}
\operatorname{comp}_{q i}=-\frac{1}{L}\left(R_{s} T_{x} V_{q s_{k-1}^{r}}^{r}-R_{s} T_{x} V_{q s_{k-1}}^{c}+L T_{x} \omega_{r} V_{d s_{k-1}}^{r}\right. \\
\left.-L T_{x} \omega_{r} V_{d s_{k-1}}^{c}\right)-\frac{V_{q s_{k-1}^{r}}^{r}-V_{q s_{k-1}}^{c}}{i-5} \\
\operatorname{comp}_{d i}=-\frac{1}{L}\left(R_{s} T_{x} V_{d s_{k-1}}^{r}-R_{s} T_{x} V_{d s_{k-1}}^{c}+L T_{x} \omega_{r} V_{q s_{k-1}^{r}}^{r}\right. \\
\left.-L T_{x} \omega_{r} V_{q s_{k-1}}^{c}\right) \\
-\frac{V_{d s_{k-1}}^{r}-V_{d s_{k-1}}^{c}}{i-5}
\end{gathered}
$$

The final equation of the oversampled compensated Deadbeat controller are the following:

$$
\begin{gathered}
{\left[\begin{array}{c}
V_{q s_{k}} \\
V_{d s_{k}}
\end{array}\right]=B_{1 T_{s_{i}}}^{-1}\left\{\left[\begin{array}{c}
i_{i_{q s_{k}}}{ }^{\text {ref }} \\
i_{d s_{k}} \\
\text { ref }
\end{array}\right]-A_{T_{s_{i}}}\left[\begin{array}{c}
i_{q s_{k}} \\
i_{d s_{k}}
\end{array}\right]-B_{2 T_{s i}}\left[\begin{array}{c}
\lambda_{m} \\
0
\end{array}\right]\right\}} \\
+\left[\begin{array}{l}
\operatorname{comp}_{q i} \\
\operatorname{comp}_{d i}
\end{array}\right]
\end{gathered}
$$

To compute the actual inverter voltages, the previous voltage references are transformed on the abc reference frame, saturated an compared with the modulator carrier. If the phase control voltage is greater or smaller than the carrier for the whole period $T_{x}$, the output phase voltage is set equal to $\frac{V_{D C}}{2}$ or $\frac{-V_{D C}}{2}$ respectively. If an intersection between the carrier and the phase voltage is present, the output phase voltage is calculated as the average voltage on the period.

\section{Simulation RESUltS}

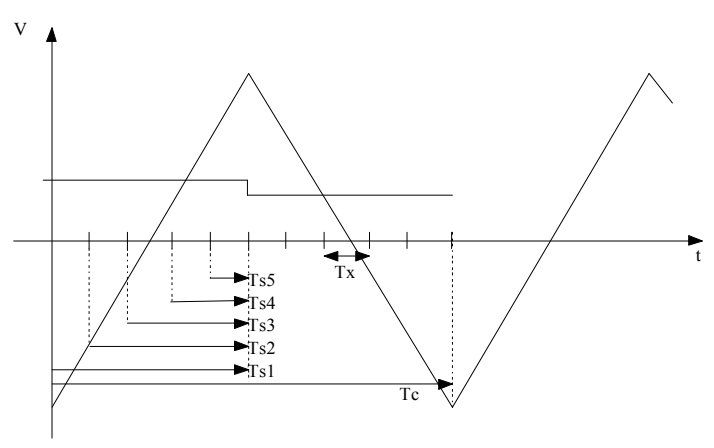

Fig. 2 - Triangular carrier, control voltage for one leg and sample times

The performance of the proposed oversampled Deadbeat algorithm has been tested in simulation using Matlab $\backslash$ Simulink. The system parameters used are listed in Table I. The results included in this paper are obtained implementing the drive with an external speed control loop that generates the reference value for the internal torque control loop. In order to verify the robustness of the proposed system to parameters uncertainties a simulative robustness analysis has been carried out. In a real application both the stator resistance and the inductance can vary. The first one is mainly affected by temperature with an uncertainty up to $30 \%$

TABLE I

SIMULATION PARAMETERS

\begin{tabular}{|c|c|c|c|}
\hline Name & Description & Value & Unit \\
\hline$R_{S}$ & Stator phase resistance & 1.5 & {$[\Omega]$} \\
\hline$L$ & Stator inductance & 2.7 & {$[\mathrm{mH}]$} \\
\hline$\lambda_{m}$ & $\begin{array}{c}\text { Flux linkage established } \\
\text { by magnets }\end{array}$ & $\begin{array}{c}0.06073 \\
3\end{array}$ & {$[\mathrm{~V} \mathrm{~s}]$} \\
\hline $\mathrm{p}$ & Pole pairs & 4 & - \\
\hline$V_{D C}$ & DC voltage source & 600 & {$[\mathrm{~V}]$} \\
\hline$T_{C}$ & Carrier period & 100 & {$[\mu \mathrm{s}]$} \\
\hline$n_{c}$ & Oversampling coefficient & 5 & - \\
\hline$T_{x}$ & Control sample time & 10 & {$[\mu \mathrm{s}]$} \\
\hline \multicolumn{4}{|r}{} \\
\end{tabular}



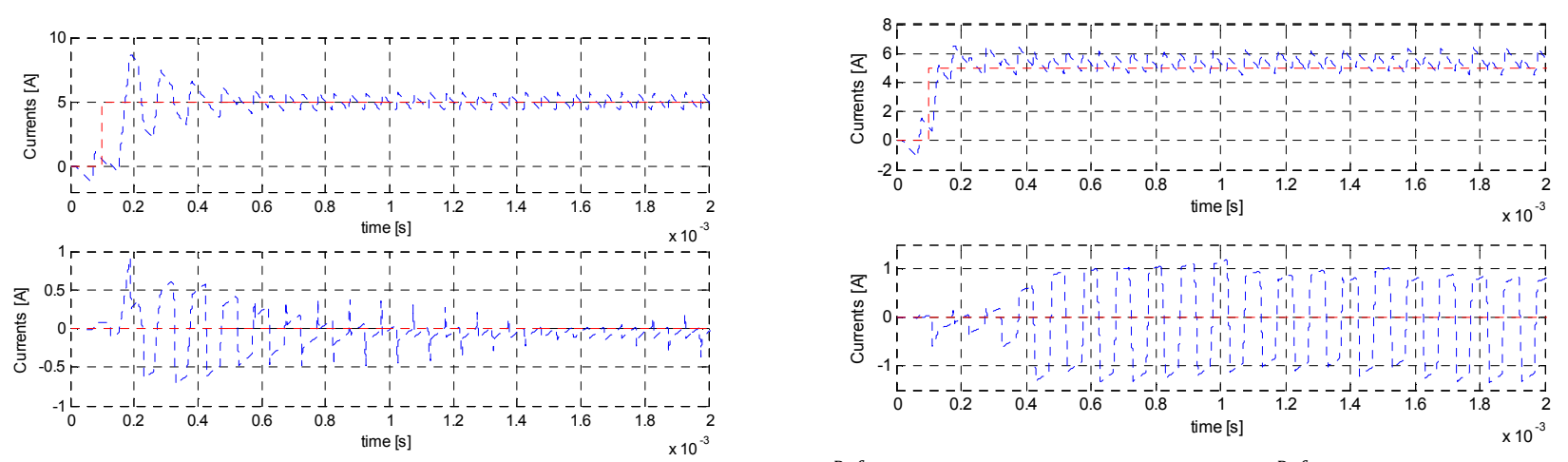

Fig. 3 - Inductance variation of 55\%. Left: Classical Deadbeat. Top: $I_{q}$ (blue) and $I_{q}^{\text {Ref }}$ (red) currents. Bottom: $I_{d}$ (blue) and $I_{d}^{\text {Ref }}$ (red) currents. Right: Deadbeat Oversampled. Top: $I_{q}$ (blue) and $I_{q}^{R e f}$ (red) currents. Bottom: $I_{d}$ (blue) and $I_{d}^{R e f}$ (red) currents.
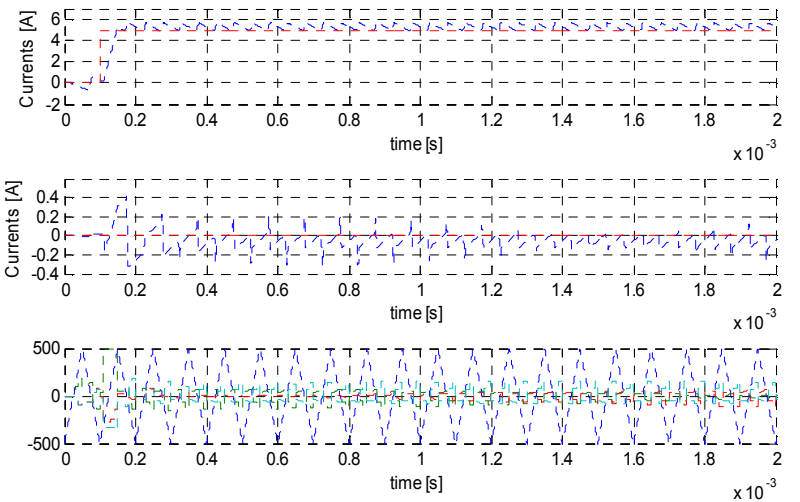

Fig. 4 - Oversample Deadbeat: Top: $I_{q}$ (blue) and $I_{q}^{\text {Ref }}$ (red) currents. Middle: $I_{d}$ (blue) and $I_{d}^{R e f}$ (red) currents. Bottom: triangular carrier and contol voltage signals
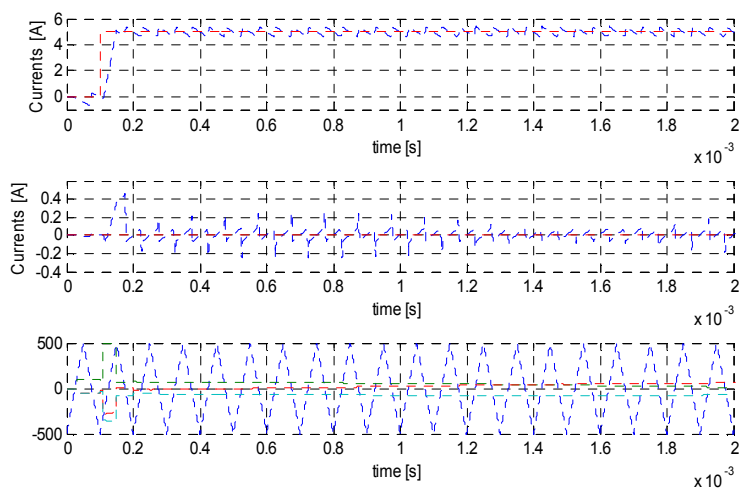

Fig. 5 - Oversampled compensated Deadbeat: Top: $I_{q}$ (blue) and $I_{q}^{\text {Ref }}$ (red) currents. Middle: $I_{d}$ (blue) and $I_{d}^{R e f}$ (red) currents. Bottom: triangular carrier and contol voltage signals

while the latter can change due to ferromagnetic saturation.

The test, whose results are reported in Fig. 3, has been therefore performed with a reduction of the machine inductance of $55 \%$ of the nominal value with respect to the one used in the controller. Fig. 3 consists in a iq current reference step from 0 to $5 \mathrm{~A}$ at $1500 \mathrm{rpm}$. It shows the
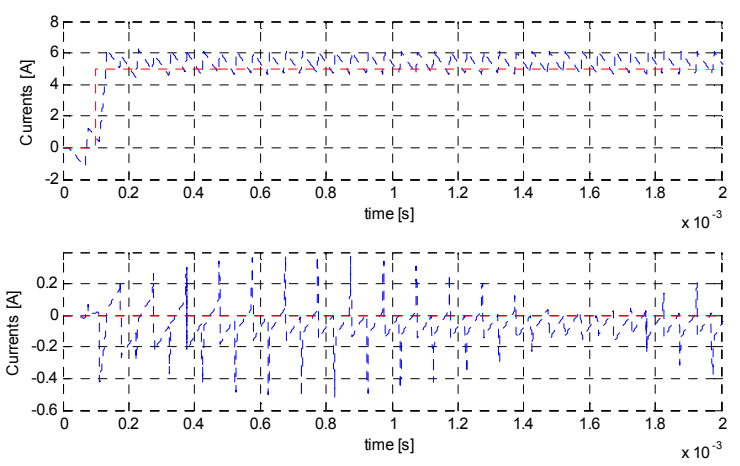

Fig. 6 - Inductance variation of $55 \%$. Oversampled compensated Deadbeat: Top: $I_{q}$ (blue) and $I_{q}^{R e f}$ (red) currents. Bottom: $I_{d}$ (blue) and $I_{d}^{\text {Ref }}$ (red) currents.

improved dynamic performance of the oversampled Deadbeat (right) compared with the classical Deadbeat (left) achieved in case of an inductance drift. It can be noticed the significant reduction in the initial current transient overshoot. The behaviour without any parameter variation is shown in Fig. 4 and Fig. 5 where the same iq current transient from 0 to $5 \mathrm{~A}$ at $1500 \mathrm{rpm}$ is proposed. It can be noticed how the introduction of the oversampling technique creates a distortion in the control voltage signals. This causes a slightly difference in the point of intersection between the carrier and the control voltages resulting in a different time of application of the same inverter configuration; as it can be seen in Fig 4 it leads to a small offset in the controlled iq and id currents. The compensation presented in the previous section has then been implemented and reported in Fig. 5 where the same test condition of Fig 4 is applied. The compensation has the advantage to eliminate the offset in the controlled currents and to remove the distortion of the control voltage signals. Fig 6 shows the case of a reduction of $55 \%$ of the nominal inductance with the oversampled compensated deadbeat control approach where a iq current transient from 0 to $5 \mathrm{~A}$ at $1500 \mathrm{rpm}$ is performed. Please notice the absence of overshoot in the response and the only offset is due to the parameter variation. The compensation does not only remove the 
controller voltages distortion but also removes the control offset introduced by the oversampling. Moreover it has been observed that comparing the classical Deadbeat $(\mathrm{Db})$, the oversampled Deadbeat (DbOs) and the compensated oversampled Deadbeat (DbOsC) that over $3000 \mathrm{rpm}$ of the rotor mechanical angular speed there is also an increase in performance due to a reduction of the phase currents THD as reported in Table II.

TABLE II

PHASE CURRENT THD [\%] FOR DIFFERENT VALUES OF ROTOR SPEED

\begin{tabular}{|c|c|c|c|}
\hline & 3000 rpm & 6000 rpm & 9000 rpm \\
\hline $\mathrm{Db}$ & 7.32 & 11.3 & 14.1 \\
\hline $\mathrm{DbOs}$ & 7.31 & 12.4 & 16.7 \\
\hline $\mathrm{DbOsC}$ & 7.33 & 11.5 & 14.8 \\
\hline
\end{tabular}

\section{CONCLUSIONS}

A new deadbeat current controller implementation is proposed in this work while preserving the main feature of the classical deadbeat control, i.e. that the control action permits, if no saturation occurs, to reach the current reference value in one sample time. The higher controller sampling frequency permits to obtain a more robust solution against parameters variation without increasing the converter switching frequency or compromise steady state performances.

\section{REFERENCES}

[1] Y. A.-R. I. Mohamed and E. F. El-Saadany, "An improved deadbeat current control scheme with a novel adaptive self-tuning load model for a three-phase PWM voltage-source inverter," IEEE Transactions on Industrial Electronics, vol. 54, pp. 747-759, 2007.

[2] S. Mariéthoz, A. Domahidi and M. Morari , "High-bandwidth explicit model predictive control of electrical drives" , IEEE Trans. Ind. Appl. , 2012 .

[3] Z. Ma, S. Saeidi and R. Kennel , "FPGA implementation of model predictive control with constant switching frequency for PMSM drives" , IEEE Trans. Ind. Informat. , vol. 10 , no. 4 , pp.2055 -2063, 2014

[4] H. L.-Huy, K. Slimani, and P. Viarouge, "Analysis and implementation of a real-time predictive current controller for permanent-magnet synchronous servo drives," IEEE Trans. Ind. Electron., vol. 41, no. 1, pp. 110-117, Feb. 1994.

[5] S. Kouro, P. Cortes, R. Vargas et al., "Model Predictive Control - A Simple and Powerful Method to Control Power Converters," IEEE Trans. Ind. Electr., vol. 56, no. 6, pp. 1826-1838, Jun. 2009.

[6] J. Rodriguez, M. P. Kazmierkowski, J. R. Espinoza et al., "State of the Art of Finite Control Set Model Predictive Control in Power Electronics," IEEE Trans. Ind. Inform., vol. 9, no. 2, pp. 1003-1016, May 2013.

[7] A. Formentini, L. de Lillo, M. Marchesoni et al., "A new mains voltage observer for PMSM drives fed by matrix converters," in EPE, 2014, pp. $1-10$.

[8] E. Fuentes, D. Kalise, J. Rodriguez et al., "Cascade-Free Predictive Speed Control for Electrical Drives," IEEE Trans. Ind. Electron., vol. 61, no. 5, pp. 2176-2184, 2014. 